American Journal of Immunology 1 (2): 68-73, 2005

ISSN 1553-619X

(c) 2005 Science Publications

\title{
Humoral Immune Response in Tuberculous Pleuritis
}

\author{
Prabha. C, Kripa V. Jalapathy and Sulochana D. Das \\ Tuberculosis Research Centre, Chennai, India
}

\begin{abstract}
Tuberculous pleuritis is a good human model to understand the local and protective immune response against tuberculosis, due to the self-limitedness of the disease. Although the cellular immune response has been well characterised in tuberculous pleurisy, much less is known about the humoral immune response operating at the site of infection. To understand the humoral immune response, B cells were enumerated in peripheral blood mononuclear cells (PBMC) and pleural fluid mononuclear cells (PFMC) of tuberculous (TP) and non-tuberculous pleuritis patients (NTP). The levels of IgG, IgA and IgM antibodies for PPD, culture filtrate (CF) and sonicate antigens (Son Ag) were assessed in plasma (BL) and pleural fluid (PF) and a western blot was carried out with the $\mathrm{CF}$ antigen. The percentage of $\mathrm{CD} 19^{+} \mathrm{B}$-cells was similar in PBMC and PFMC of TP patients but was significantly lower in PFMCs of NTP patients. The IgG levels for PPD and CF antigens were higher in PF of TP than NTP patients. The antigen recognition patterns did not differ in plasma and pleural fluid of the same patient in both groups pointing out the passive diffusion of the plasma to the pleura. The antigens $25,31,33,70,110,124$ and $132 \mathrm{kDa}$ were recognized exclusively by the TP patients. Thus our study showed that the local humoral response in TP did not differ from the systemic response. However, the humoral response differed in TP patients when compared to NTP patients.
\end{abstract}

Key words: Tuberculous pleuritis, humoral response, mycobacterial antigens

\section{INTRODUCTION}

Tuberculosis is the leading cause of death and continues to be the foremost cause of pleural effusion ${ }^{[1]}$. Of all the extra pulmonary manifestation of tuberculosis, tuberculous pleuritis (TP) is predominant and is a pauci-bacillary disease occurring due to the delayed type hypersensitive response to the mycobacterial antigens. Immunological reactivity against Mycobacterium tuberculosis is compartmentalized in TP and is often characterized by resolution of the disease without chemotherapy ${ }^{[2,3]}$. The compartmentalized immune response in TP allows it to be exploited as a human model to understand the local and protective host immune functions.

The different antigenic components of $M$. tuberculosis have the innate ability to trigger adaptive immune response in the host. It is well known that in tuberculosis, cell mediated immune response (CMI) is host-protective. The mycobacterial antigens that are good enough to elicit CMI are also equally effective in evoking antibody mediated humoral immune response. The current understanding of the humoral immune response to tuberculosis is from the serological studies that by and large aimed at developing a serodiagnostic test to identify tuberculosis. Though these studies provide information on the pattern of antibody response at the systemic level, much lesser is known about the antibody response at the site of infection.
The genomic studies on $M$. tuberculosis have predicted 3924 protein-coding sequences (CDS) from the genome ${ }^{[4]}$. The recent study by Mawuenyega et al has characterized 1044 protein and their corresponding localizations with respect to cell wall, membrane and cytoplasm by high-throughput proteomics and computational approaches ${ }^{[5]}$. To date, in the Tuberculist genome analysis worldwide web server, there are 113 proteins annotated as secreted and 49 , as excreted proteins of M.tuberculosis ${ }^{[4]}$. The exported and secreted proteins of mycobacteria are hypothesized, by several researchers as the key protective antigens ${ }^{[6-10]}$. This is due to the observations that live but not dead bacilli induce a protective T-cell response and that the cell surface and secreted antigens are the first encountered and presented by the host immune response. In addition to the protective T-cell response, the secreted proteins are also recognized by humoral arm of the immune response. The most studied secreted proteins of $M$. tuberculosis are ESAT-6 (early secretory antigenic target), CFP10, 38kDa, $16 \mathrm{kDa}$ and antigen 85 complex $^{[11-16]}$. The ability of these proteins to elicit serological response make them to be utilized as the candidates for sero-diagnosis.

The knowledge of antibody response at the site of infection may possibly help us in identifying newer antigens that are immunogenic and crucial for provoking host antibody response or that may aid in sero-diagnosis. Hence, in the present study we aimed to

Corresponding Author: Dr. Sulochana, D. Das, Sr. Research Officer, Department of Immunology, Tuberculosis Research Centre, Mayor V.R. Ramanathan Road, Chetput, Chennai 600 031, Tel: 91-44-2836 9677, Fax: 91-44-2836 2528 
evaluate the humoral immune response in TB pleuritis i.e at the site of infection. Hence, we assessed the levels of different antibodies against different mycobacterial antigens in both plasma and pleural fluid of the same patient by ELISA. Also we compared the localized and systemic antibody response against the secreted antigens by western blot analysis.

\section{SUBJECTS AND METHODS}

Study Group: In this study, we enrolled a total of 74 patients with pleural effusion from Government General Hospital. The study was approved by the institutional ethical committee of Government General Hospital, Chennai. The written and informed consent was obtained from each study participant. Patients with HIV infection were excluded from the study. The blood and the pleural fluid collected for the therapeutic purpose, before the start of the treatment was utilized for this study. All these subjects were Mantoux positive and showed an induration of more than $10 \mathrm{~mm}$. Based on the diagnosis, the study subjects were classified into two groups.

Tuberculous pleuritis subjects (TP): This group consists of 44 patients, who had pleural effusion due to tuberculous infection. These subjects were sputum smear positive for the acid-fast bacilli and were Mantoux positive. Further diagnosis of pleural tuberculosis was based on positive culture of $M$. tuberculosis on Lowenstein-Jenson medium from pleural fluid and by PCR to detect the bacilli using IS6110 primers in pleural fluid, supported by the radiographic and clinical data.

Non-tuberculous pleuritis subjects (NTP): This group consists of 30 subjects who had exudative pleural effusion due to causes other than tuberculosis. The etiologies of these patients are Malignancy $(\mathrm{N}=24)$, Para pneumonic effusion $(\mathrm{N}=4)$ and exudative effusion due to secondary infections to autoimmune disorders $(\mathrm{N}=2)$. The effusion had lymphoctyic predominance. The diagnosis was based on the cytology of the pleural fluid, demonstration of the causative organism by appropriate culture methods and relevant clinical findings.

Antigens: Purified Protein Derivative was obtained from Central Veterinary Lab, Weybridge, U.K.

Culture filtrate antigen was prepared from $M$. tuberculosis H37Rv grown in Sauton's liquid medium for 5 weeks. For the preparation of culture filtrate, the proteins in the culture supernatant were precipitated with $90 \%$ ammonium sulphate saturation. The precipitate was dissolved and dialyzed against $0.1 \mathrm{M}$ PBS.
The sonicate antigen was prepared by sonicating the pellet containing bacilli obtained after removing the culture supernatant. The sonication was carried out in 6 cycles of each 30 seconds on and 30 seconds off in PBS ( $\mathrm{pH}$ 7.0) followed by centrifugation at $8000 \mathrm{rpm}$ for $10 \mathrm{~min}$. The supernatant was filtered through $0.45 \mu \mathrm{m}$ pore size membrane.

The quantification of protein was done using the protein estimation kit following manufacturer's instruction (Bangalore Genei, India)

Flow cytometry: The percentage of B-cells in the peripheral blood mononuclear cells (PBMC) and pleural fluid mononuclear cells (PFMC) were assessed by flow cytometry. The cells were incubated with CD3 FITC/ CD19 PE antibodies for $30 \mathrm{~min}$ at $4^{0} \mathrm{C}$. The cells were fixed in $1 \%$ paraformaldehyde (W/V) and acquired using fluorescence-activated cell scan (FACS, Becton Dickinson). The analyses were done using CellQuest software. A total of 10, 000 events for each sample were acquired. The data were expressed as percentage of total lymphocytes.

ELISA: ELISA was carried out to estimate the immunoglobulins $\operatorname{IgG}$, IgA and IgM levels against PPD, culture filtrate (CF) and sonicate antigens (Son $\mathrm{Ag})$. The antigens were coated on the polyvinyl microtitre ELISA plates (Costar, USA) at the concentration of $5 \mu \mathrm{g} / \mathrm{ml}$. After overnight incubation, non-specific adsorption of protein was blocked with $1 \% \mathrm{BSA}$ in PBS for one hour at $37^{\circ} \mathrm{C}$. The plasma and pleural fluid samples were added in 1:100 dilutions in triplicates. Peroxidase-labeled Goat anti-human IgG, IgA and IgM were used at the dilutions of 1:10,000, 1: 5000 and 1: 10,000 respectively. The enzymatic substrate used was $\mathrm{H}_{2} \mathrm{O}_{2}$ mixed with the chromogen substrate tetramethylbenzidine (Bangalore Genei, India). The absorbance was read at $450 \mathrm{~nm}$ using an ELISA plate reader (Molecular devices, SPECTRA MAX-250, USA).

Western blotting: The culture filtrate antigen was denatured under reducing condition at $100^{\circ} \mathrm{C}$ in a sand bath for 5 minutes and separated by SDS-PAGE on $12 \%$ gel. The fractionated proteins were electrophoretically transferred onto PVDF membrane (Bio Rad) in a transblot unit (Transblot, BioRad). Membranes were blocked with $1 \%$ BSA in PBS containing $0.1 \%$ tween-20. The membrane was cut into $5-7 \mathrm{~mm}$ wide strips and then incubated with plasma and pleural fluid samples in 1:100 dilution. Peroxidase labeled goat-anti-human $\operatorname{IgG}$ was used as a secondary antibody in 1:5000 dilution. The binding of the antibodies to the specific protein was revealed by using Diaminobenzidine (DAB) chromogen as substrate. 
Table 1a: Antibody levels in tuberculous pleuritis subjects $(\mathrm{N}=44)$

\begin{tabular}{lllllll}
\hline & PPD & & CF & & Son Ag & \\
\hline & BL & PF & BL & PF & BL & PF \\
\hline IgG & $1.2 \pm 0.1$ & $1 \pm 0.1$ & $1.6 \pm 0.12$ & $1.4 \pm 0.12^{*}$ & $0.7 \pm 0.09$ & $0.7 \pm 0.1$ \\
IgA & $0.5 \pm 0.05$ & $0.4 \pm 0.06$ & $0.4 \pm 0.04$ & $0.4 \pm 0.05$ & $0.50 \pm 0.03$ & $0.50 \pm 0.04$ \\
IgM & $0.3 \pm 0.05$ & $0.26 \pm 0.04 *$ & $0.4 \pm 0.05$ & $0.36 \pm 0.04$ & $0.6 \pm 0.06$ & $0.5 \pm 0.05$ \\
\hline
\end{tabular}

Table 1b: Antibody levels in non-tuberculous pleuritis subjects $(\mathrm{N}=30)$

\begin{tabular}{lllllll}
\hline & PPD & \multicolumn{3}{l}{ Son Ag } \\
\hline & BL & PF & BL & PF & BL & PF \\
\hline IgG & $1.0 \pm 0.09$ & $0.81 \pm 0.08^{* *}$ & $1.32 \pm 0.1$ & $1 \pm 0.09^{*}$ & $0.9 \pm 0.1$ & $0.7 \pm 0.08^{*}$ \\
IgA & $0.6 \pm 0.07$ & $0.4 \pm 0.06^{*}$ & $0.5 \pm 0.06$ & $0.3 \pm 0.05^{*}$ & $0.50 \pm 0.05$ & $0.4 \pm 0.06^{*}$ \\
IgM & $0.4 \pm 0.04$ & $0.3 \pm 0.06^{*}$ & $0.4 \pm 0.05$ & $0.3 \pm 0.05^{*}$ & $0.6 \pm 0.06$ & $0.4 \pm 0.06^{*}$ \\
\hline
\end{tabular}

The antibody isotype levels in plasma (BL) and pleural fluid (PF) of 44 tuberculous (A) and 30 non-tuberculous pleuritis subjects (B) for PPD, culture filtrate (CF) and sonicate antigens (Son Ag) of M. tuberculosis $\mathrm{H} 37 \mathrm{Rv}$ as assessed by ELISA. The coating concentration of the antigens was $5 \mu \mathrm{g} / \mathrm{ml}$ and the optical density (O.D.) was measured at $450 \mathrm{~nm}$.

* Represents $\mathrm{p}<0.05$ in comparison with the corresponding plasma

\# Represents $\mathrm{p}<0.05$ for comparison between TP and NTP groups

A pre-stained molecular weight marker (GIBCO BRL) was run on the same gel.

Statistical analysis: The significance of the observed differences was calculated using paired or unpaired student's t-test as appropriate.

\section{RESULTS}

To understand the in vivo humoral response, the percentage composition of B-cells in PBMC and PFMC was determined. There was no significant difference in the levels of $\mathrm{CD} 19^{+} \mathrm{B}$-cells between PBMC and PFMC of TP patients. The $\mathrm{CD} 19^{+} \mathrm{B}$-cells were significantly lower in the PFMCs of non-tuberculous pleuritis patients when compared to their PBMCs (Fig. 1).

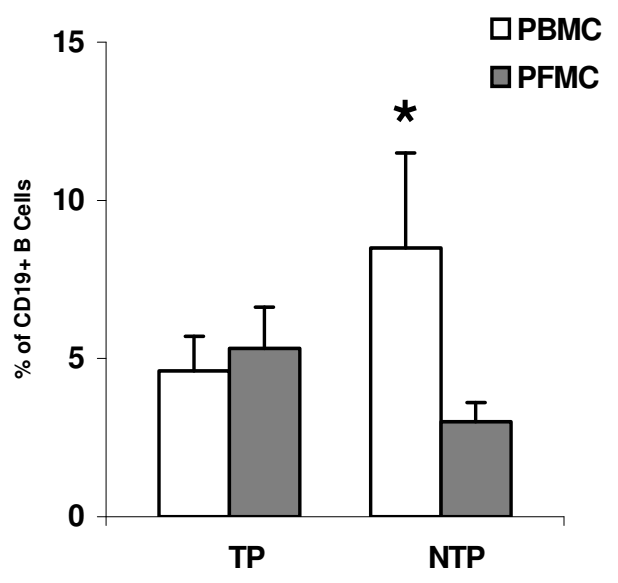

Fig.1: Percentage of B-cells in PBMC and PFMC in 44 TP and 30 NTP patients: The percentage of B-cells was determined using a double staining protocol by FACS. The mean levels $( \pm$ S.E.M.) are represented in the graph. $*$ Represents $\mathrm{p}<0.05$

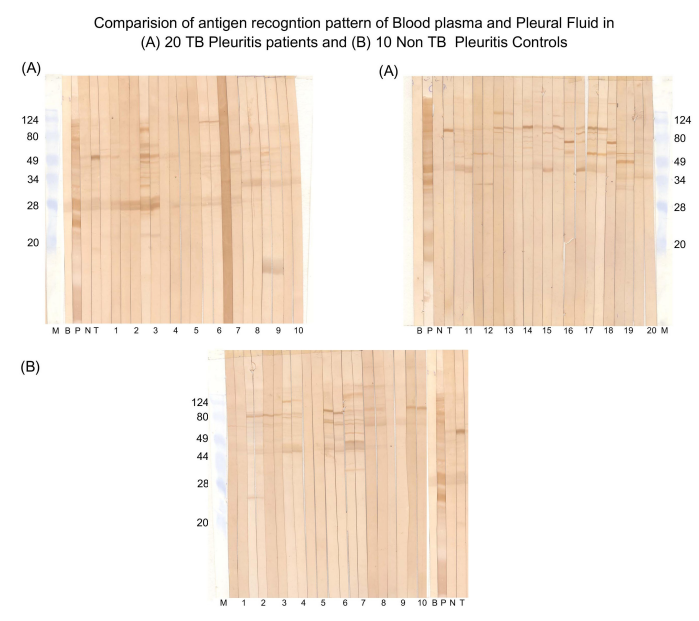

Fig. 2: Western blot analysis of culture filtrate antigen of H37Rv: The band patterns recognised by plasma and pleural fluid of 20 tuberculous pleuritis subjects (A-lanes 1 to 20) and 10 nontuberculous pleuritis control subjects (B-lanes 1 to 10). Molecular weight markers in kilodaltons $(\mathrm{kDa})$ are indicated on the left $(\mathrm{M})$. B-blank, P-pooled TB plasma, N-pooled normal plasma, T-pooled tuberculous pleuritis plasma.

The systemic levels of $\mathrm{CD} 19^{+} \mathrm{B}$-cells in NTP were higher than in TP patients. The levels of antibodies did not differ significantly between plasma and pleural fluid of TP patients except for IgG levels against CF antigens and IgM levels against PPD, where the antibody levels were significantly lower in pleural fluid when compared with plasma (Table 1a). Interestingly in non-tuberculous pleuritis patients, the antibody levels in the pleural fluid against all the mycobacterial antigens were significantly lower than the plasma antibody levels (Table 1b). The IgG levels were higher in PF of 
Am. J. Immunology 1 (2): 68-73, 2005

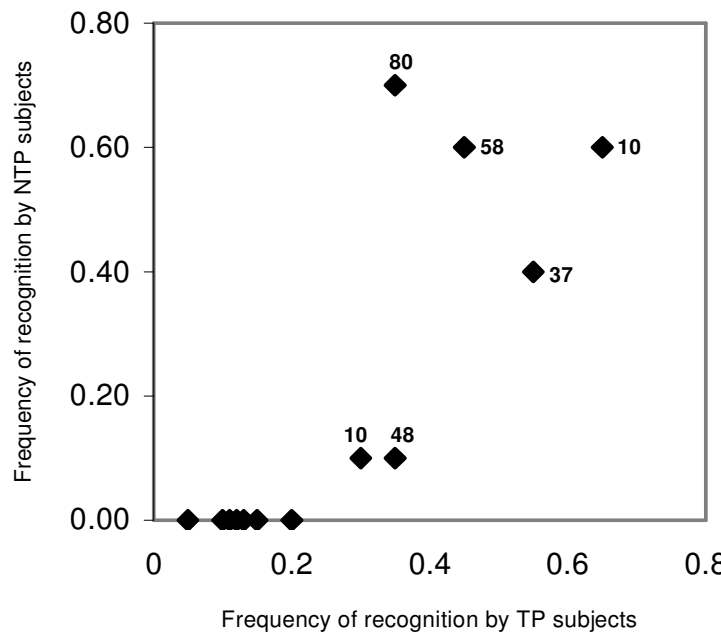

Fig. 3: Graphical analysis of the antibody reactivity patterns as observed in figure 2: The frequency of reaction of the plasma of tuberculous pleuritis subjects (TP) with a given antigen band is plotted against frequency with nontuberculous pleuritis subjects (NTP). The apparent molecular weight is indicated alongside. The dots lying on $\mathrm{x}$-axis represents the molecular weights of $25,33,124,110,70$, 31 and $132 \mathrm{kDa}$ respectively. These antigens were recognised only by TP subjects

TP than NTP subjects for PPD and CF antigens. The IgA and IgM antibody levels did not differ in the plasma of the two groups for all the antigens (Table 1a and $b$ ).

There was a differential recognition of the culture filtrate antigens of mycobacteria between TP and NonTP patients by western blot. However there was no difference in the antigen recognition patterns of plasma and pleural fluid of the same patients in both the groups (Fig. 2a and b). Some of the antigens were recognised in both TP and NTP subjects and some were exclusively recognized by TP plasma and pleural fluid. The antigens of molecular weight $25,31,33,70,110$, 124 and $132 \mathrm{kDa}$ were recognized exclusively by the TP patients. The frequency of recognition of some of the culture filtrate antigens on an immunoblot is shown in Fig. 3.

\section{DISCUSSION}

Pleural tuberculosis is a self-limiting illness. The fact that the recurrent pleurisy is rare indicates that there occurs the adequate local and protective immune response. The successful self-elimination of the condition is brought out by the compartmentalized $\mathrm{CD} 4^{+} \mathrm{Th} 1$ immune response in $\mathrm{TP}^{[2,17]}$. Although the inevitable role of the $\mathrm{CD}^{+} \mathrm{T}$ cells and the Th1 cytokines in limiting the illness is well documented, much less is known about the contribution of B-cell mediated immunity in tuberculous pleuritis ${ }^{[18,19]}$.
The absence of any significant difference in the number of B-cells between the pleural fluid and blood in TP, observed in the present study indicates the absence of compartmentalization of these cells. A similar observation was reported by Santiago et al. ${ }^{[20]}$. These results show that there is no quantitative difference in the B-cell mediated response. However, the report by Wallis et al showed that the tuberculous pleural fluid contains a high proportion of antigen reactive $\mathrm{B}-\mathrm{cells}^{[21]}$. Also they had generated the clones of B-cells from pleural fluid that were reactive to specific antigens in the range from $29 \mathrm{kDa}$ to $68 \mathrm{kDa}$. This indicates that there occurs mycobacterial antigen specific B-cell clonal propagation at the site of infection and it is important to analyze them for further development in vaccine and/or diagnosis.

In our study, though the IgG levels for PPD were significantly higher in PF of TP than NTP subjects, the levels of other isotype of antibodies did not differ. Banchuin $e t$ al have reported the absence of significant difference in the IgG levels for PPD in pleural fluid between patients with tuberculosis and malignancy ${ }^{[22]}$. A contrary report by Murate et al, demonstrated a significant increase of all the three isotypes ( $\operatorname{IgA}, \operatorname{IgG}$ and $\operatorname{IgM}$ ) for PPD in the tuberculous pleural effusion than the malignant pleurisy ${ }^{[23]}$.

There are very few reports on the pleural antibody response to secretary antigens of mycobacteria. Most of these studies have documented the usefulness of a single or the combination of purified secretary antigens of mycobacteria in serological or pleural fluid diagnosis ${ }^{[24-26]}$. And there are no reports that compare the systemic verses local antibody response to the secretary antigens and its usefulness in discriminating the non-tuberculous pleuritis subjects. Our results indicate that the local antibody production, against the culture filtrate antigens does not differ significantly from that of the systemic levels. Nevertheless the differential antigen recognition on western blot by TP and NTP subjects supports the suggestion of Wallis et al., ${ }^{[21]}$, that tuberculous pleurisy may prove useful in the identification of potentially protective mycobacterial antigens particularly those secreted during infection. Of particular interest from our study is the low Mol.wt. proteins of 31 and $33 \mathrm{kDa}$ which were recognized only by the TP subjects as also reported by Wallis et al., ${ }^{[21]}$. The larger proportion of patients recognized the antigens in the high molecular weight region of $70-130 \mathrm{kDa}$, particularly $70,110,124$ and $132 \mathrm{kDa}$ proteins. It suggests that these secreted antigens of M. tuberculosis, elicit antibodies in majority of TP patients. A similar observation was reported by Laal et al in patients with pulmonary tuberculosis ${ }^{[27]}$. This shows that the antigens of high molecular weight have an important role in the protective immune response. Characterization of such proteins is essential to understand their role in pathogenesis of tuberculosis. 
In the present study, the individual antibody response of isotypes $\operatorname{IgA}$ and $\operatorname{IgM}$ to the mycobacterial antigens did not show any appreciable difference between the TP and NTP subjects. Also NTP subjects recognized some of the secretary antigens of mycobacteria. This might be due to the high prevalence of tuberculosis in our country and all of the non-TP subjects recruited in the study are infected with mycobacteria as evident from their mantoux status. This is supported by the fact that the anti-mycobacterial antibody detection has modest diagnostic value for tuberculosis and tuberculous pleuritis in area with intermediate or high prevalence of tuberculosis ${ }^{[26]}$.

Together our data suggests that the antibodies present in the pleural fluid might probably be due to the passive diffusion from the plasma rather than the local production as reported previously ${ }^{[28]}$. However studies that employed single or multiple, purified or recombinant antigens support the concept of the local antibody production in TP pleuritis ${ }^{[21,26]}$. Such studies employing purified antigens have to be carried out particularly in an endemic area to ascertain whether there is local antibody production against specific antigens of mycobacteria. Thus our study demonstrates that the local humoral anti-mycobacterial response does not differ extensively from the systemic humoral immune response in tuberculous pleuritis.

\section{ACKNOWLEDGEMENT}

Prabha. C and Kripa. V. Jalapathy render their thanks to Indian Council of Medical Research (I.C.M.R) and Council of Scientific and Industrial Research (C.S.I.R) respectively, for providing Senior Research fellowships.

\section{REFERENCES}

1. Ferrer, J., 1997. Pleural tuberculosis. Eur. Respir. J., 10: 942-947.

2. Barnes, P.F., S.D. Mistry, C.L. Cooper, C. Pirmez, T.H. Rea and R.L. Modlin, 1989. Compartmentalization of a CD4+ T lymphocyte subpopulation in tuberculous pleuritis. J. Immunol., 142: 1114-9.

3. San Jose, M.E., L. Valdes, M.J. Saavedra, J.M. De Vega, D. Alvarez, J. Vinuela, P. Penela, J M. Valle and R. Seoane, 1999. Lymphocyte populations in tuberculous pleural effusions. Ann. Clin. Biochem., 36: 492-500.

4 http://genolist.pasteur.fr/TubercuList/genome.cgi.

5. Mawuenyega, K.G., C.V. Forst, K.M. Dobos, J.T. Belisle, J. Chen, E.M. Bradbury, A R. Bradbury and X. Chen, 2005. Mycobacterium tuberculosis functional network analysis by global subcellular protein profiling. Mol. Biol. Cell., 16: 396-404.
6. Andersen, P., 1994. Effective vaccination of mice against Mycobacterium tuberculosis infection with a soluble mixture of secreted mycobacterial proteins. Infect. Immun., 62: 2536-44.

7. Hubbard, R.D., C.M. Flory and F.M. Collins, 1992. Immunization of mice with mycobacterial culture filtrate proteins. Clin. Exp. Immunol., 87: 94-8.

8. Pal, P.G. and M.A. Horwitz, 1992. Immunization with extracellular proteins of Mycobacterium tuberculosis induces cell-mediated immune responses and substantial protective immunity in a guinea pig model of pulmonary tuberculosis. Infect. Immun., 60: 4781-92.

9. Roberts, A.D., M.G. Sonnenberg, D.J. Ordway, S.K. Furney, P.J. Brennan J.T. Belisle and I.M. Orme, 1995. Characteristics of protective immunity engendered by vaccination of mice with purified culture filtrate protein antigens of Mycobacterium tuberculosis. Immunology, 85: 502-8.

10. Orme, I.M., P. Andersen and W.H. Boom, 1993. T cell response to Mycobacterium tuberculosis. J. Infect. Dis., 167: 1481-97.

11. Sorenson, A.L., N.S.G. Houen, P. Andersen and A.B. Andersen, 1995. Purification and characterization of a low molecular- mass T-cell antigen secreted by Mycobacterium tuberculosis. Infect. Immun., 63:1710-1717.

12. Wilkinson, K.A., R.J. Wilkinson, A. Pathan, K. Ewer, M. Prakash, P. Klenerman, N. Maskell, R. Davies, G. Pasvol and A. Lalvani, 2005. Ex vivo characterization of early secretory antigenic target 6-specific $\mathrm{T}$ cells at sites of active disease in pleural tuberculosis. Clin. Infect. Dis., 40: 184-7.

13. Nicol, M.P., D. Pienaar, K. Wood, B. Eley, R.J. Wilkinson, H. Henderson, L. Smith, S. Samodien and D. Beatty, 2005. Enzyme-linked immunospot assay responses to early secretory antigenic target 6 , culture filtrate protein 10 , and purified protein derivative among children with tuberculosis: implications for diagnosis and monitoring of therapy. Clin. Infect. Dis., 40: 1301-8.

14. van Pinxteren, L.A., P. Ravn, E.M. Agger, J. Pollock and P. Andersen, 2000. Diagnosis of tuberculosis based on the two specific antigens ESAT-6 and CFP10. Clin. Diagn. Lab. Immunol., 7: $155-60$.

15. Senthil, Kumar, K.S., K.R. Uma Devi and R. Alamelu, 2002. Isolation and evaluation of diagnostic value of two major secreted proteins of Mycobacterium tuberculosis. Indian J. Chest Dis. Allied Sci., 44: 225-32.

16. Uma Devi, K.R., B. Ramalingam and A. Raja, 2003. Antibody response to Mycobacterium tuberculosis 30 and $16 \mathrm{kDa}$ antigens in pulmonary tuberculosis with human immunodeficiency virus coinfection. Diagn. Microbiol. Infect. Dis., 46: 205-9. 
17. Jalapathy, K.V., C. Prabha and S.D. Das, 2004. Correlates of protective immune response in tuberculous pleuritis. FEMS Immunol. Med. Microbiol., 40: 139-45.

18. Barnes, P.F., S. Lu, J.S. Abrams, E. Wang, M. Yamamura and R.L. Modlin, 1993. Cytokine production at the site of disease in human tuberculosis. Infect. Immun., 61: 3482-9.

19. Aoe, K., A. Hiraki, T. Murakami, K. Murakami, K. Makihata, K. Takao, R. Eda, T. Maeda, K. Sugi, Z. Darzynkiewicz and H. Takeyama, 2003. Relative abundance and patterns of correlation among six cytokines in pleural fluid measured by cytometric bead array. Intl. J. Mol. Med., 12: 193-8.

20. Montes Santiago, J., F. Gambon Deza, M. Pacheco Carracedo and T. Cerda Mota, 1996. Cellular immune response in tuberculosis: analysis of $\mathrm{T}$ lymphocytes and their subsets, B-lymphocytes and natural cytotoxic cells in different tuberculosis states and body fluids. Rev. Clin. Esp., 196: 223-7.

21. Wallis, R.S., S.L. Alde, D.V. Havlir, M.H. AmirTahmasseb, T.M. Daniel and J.J. Ellner, 1989. Identification of antigens of Mycobacterium tuberculosis using human monoclonal antibodies. J. Clin. Invest., 84: 214-9.

22. Banchuin, N., U. Pumprueg, V. Pimolpan, S. Sarasombath and K. Danpukdee, 1987. Anti-PPD IgG responses in tuberculous pleurisy. J. Med. Assoc. Thai., 70: 321-5.
23. Murate, T., K. Mizoguchi, H. Amano, K. Shimokata and T. Matsuda, 1990. Antipurifiedprotein-derivative antibody in tuberculous pleural effusions. Chest, 97: 670-3.

24. Gupta, S., S. Kumari, J.N. Banwalikar and S.K. Gupta, 1995. Diagnostic utility of the estimation of mycobacterial Antigen A60 specific immunoglobulins $\operatorname{IgM}$, IgA and IgG in the sera of cases of adult human tuberculosis. Tuber Lung Dis., 76: 418-24.

25. Kunter, E., K. Cerrahoglu, A. Ilvan, T. Isitmangil, O. Turken, O. Okutan, Z. Kartaloglu and S. Cavuslu, 2003. The value of pleural fluid anti-A60 IgM in BCG-vaccinated tuberculous pleurisy patients. Clin. Microbiol. Infect., 9: 212-20.

26. Chierakul, N., P. Damrongchokpipat, A. Chaiprasert and W. Arjratanakul, 2001. Antibody detection for the diagnosis of tuberculous pleuritis. Intl. J. Tuberc. Lung Dis., 5: 968-72.

27. Laal, S., K.M. Samanich, M.G. Sonnenberg, S. Zolla-Pazner, J.M. Phadtare and J.T. Belisle, 1997. Human humoral responses to antigens of Mycobacterium tuberculosis: Immunodominance of high-molecular-mass antigens. Clin. Diagn. Lab. Immunol., 4: 49-56.

28. Levy, H., L.G. Wayne, B.E. Anderson, P.F. Barnes, and R.W. Light, 1990. Antimycobacterial antibody levels in pleural fluid as reflection of passive diffusion from serum. Chest, 97: 1144-7. 